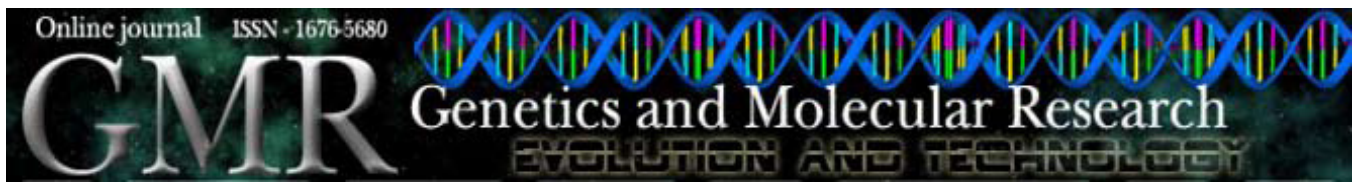

\title{
Pattern of silver nitrate-staining during meiosis and spermiogenesis in testicular lobes of Antiteuchus tripterus (Heteroptera: Pentatomidae)
}

\author{
H.V. Souza, M.M.U. Castanhole, H.E.M.C. Bicudo and M.M. Itoyama \\ Laboratório de Citogenética de Insetos, Departamento de Biologia, \\ Instituto de Biociências, Letras e Ciências Exatas, \\ Universidade Estadual Paulista, São José do Rio Preto, SP, Brasil \\ Corresponding author: M.M. Itoyama \\ E-mail: mary@ibilce.unesp.br
}

Genet. Mol. Res. 7 (1): 196-206 (2008)

Received November 10, 2007

Accepted December 11, 2007

Published January 26, 2008

\begin{abstract}
The pattern of silver nitrate (Ag)-staining differed among testicular lobes of Antiteuchus tripterus. In general, these differences are in regard to the number, size, shape, coloring intensity, and location of the stained bodies or masses, observed during meiosis and spermiogenesis. These characteristics were similar in lobes 1-3. Lobes 4-6, however, differed from each other and from lobes 1-3 as well. Because the Ag-staining method is specific for nucleolar organizing regions and nucleolar material, the observations in meiosis of lobes 1-3 suggested the presence of a single pair of nucleolar organizing region-bearing chromosomes in A. tripterus, as previously found in other Pentatomidae species. In general, the amount of Ag-stained material seen in meiosis of the testicular lobes 1-3 of A. tripterus is smaller than in the other lobes. The differences among
\end{abstract}


lobes observed during spermiogenesis included a striking variation in morphology of the Ag-stained material found in the head and tail of the spermatids. Given that the key role of the nucleolar material is to participate in protein synthesis, interlobular variations seem to be related to the different functions attributed to each lobe (reproduction to lobes 1-3 and basically nutrition to lobes 4-6). To our knowledge, this is the first time that the nucleolar material was studied in each testicular lobe during spermatogenesis. The present observations encourage further studies since, in addition to being of basic biological interest, several Pentatomidae species are agricultural pests and added knowledge of their biology, mainly in reproduction, may be important for the development of control strategies.

Key words: Heteroptera; Pentatomidae; Antiteuchus; Nucleolus; Testicular lobe differentiation

\section{INTRODUCTION}

In most arthropod species, nucleoli dissociate at either diplotene or diakinesis. Therefore, small bodies impregnated by the silver nitrate (Ag) technique, which is specifically used to stain proteins that bind to ribosomal RNA (rRNA), cannot be seen from metaphase to telophase I. As spermatids begin to form, nucleolar bodies reappear, indicating resumption of rRNA transcription, and finally disappear at the end of spermatid development (Bressa et al., 2003). However, some exceptions have been reported. In Asellus aquaticus (Isopoda), Ag-nucleolar organizing region (NOR) bodies have been visualized throughout spermatogenesis (Di Castro et al., 1983). In Callicrania seanei (Orthroptera), not only NORs have been observed during both divisions, but also nucleolar bodies scattered among chromosomes during interkinesis and even pro-metaphase II (Santos et al., 1987). In Triatoma infestans and T. sordida (Heteroptera, Reduvidae), Ag-NOR-stained masses have been observed up to metaphase I (Tavares and Azeredo-Oliveira, 1997). In Carlisis wahlbergi (Heteroptera, Coreidae), nucleolar semi-persistence (presence of nucleolar bodies in metaphases) has been reported up to metaphase II (Fossey and Liebenberg, 1995), while in Acanthocoris sordidus (Heteroptera, Coreidae) and Coptosoma punctissimum (Heteroptera, Plataspidae), nucleoli have been detected in metaphase plates of both primary and secondary spermatocytes (Yoshida, 1947; Bressa et al., 2003).

In the present study, nucleolar activity was assessed in the testes of Antiteuchus tripterus (Heteroptera, Pentatomidae). As described by Souza et al. (2007) this species has six testicular lobes (numbered 1 through 6). Lobe 5 contains lobe 6 in its interior and is, therefore, a compound lobe. Using the stain lacto-acetic orcein, the same authors observed that in lobes 1 through 3, meiosis is the same as in other Heteroptera species previously described. In lobe 4, however, the morphology of meiotic prophase cells is characteristic of the so-called "diffuse" stage. In this lobe, meiotic cells are always larger than those in lobes 1-3 displaying a region of peculiar "pleated" appearance in the posterior region of the spermatid head. Lobe 5 is called "harlequin" because it forms spermatozoa of different sizes. Lobe 6, contained by lobe 5, shows prophase cells, also at a "diffuse"-like stage, 
larger than in lobe 5 ; in lobe 6 there are cross-partitions in the posterior region of the head and anterior region of the tail, which are not found in any of the other lobes (Souza et al., 2007).

In the present study, using silver nitrate technique, variations in the morphology and positioning of the nucleolar material were analyzed in the six testicular lobes of $A$. tripterus during meiosis and spermiogenesis. The aim was to provide further information on cytogenetic differentiation of these testicular structures in meiosis and spermiogenesis of Heteroptera species.

\section{MATERIAL AND METHODS}

Insects were collected in São José do Rio Preto, SP, Brazil, and dissected in Demerec physiological solution (Demerec and Kaufmann, 1945). The lobes of the seminiferous tubules were isolated according to the technique described by Souza et al. (2007). Testes were incubated for 20 min after adding a drop of trypsin and EDTA solution (0.005 $\mathrm{g}$ trypsin and $0.002 \mathrm{~g}$ EDTA diluted in $10 \mathrm{~mL}$ Hank's solution $(1.4 \mathrm{mM} \mathrm{NaCl}, 70 \mathrm{mM}$ $\mathrm{KCl}, 4.2 \mathrm{mM} \mathrm{Na}_{2} \mathrm{HPO}_{4}, 3 \mathrm{mM} \mathrm{KH} \mathrm{PO}_{4}, 5 \mathrm{mM}$ glucose, $0.045 \mathrm{mM}$ phenol red), and the lobes were then separated, placed on different slides, fixed and stained with silver nitrate according to Howell and Black (1980). This technique specifically stains proteins that associate with rRNA following transcription (Howell et al., 1975; Goodpasture and Bloom, 1975; Miller et al., 1977). The images that illustrate this study were obtained under an AXIOSKOP 2 ZEISS light microscope (AXIO VISION software) in the Morphology Laboratory of the Department of Biology, IBILCE/UNESP, São José do Rio Preto, SP.

\section{RESULTS}

The assessment of the characteristics of silver-impregnated nucleolar material, in each of the six testicular lobes of adult Antiteuchus tripterus specimens, showed that in lobes 1-3 polyploid nuclei of the nutritive cells exhibit numerous silver-impregnated nucleolar granules, some of them fused together (Figure 1a), while spermatogonial cells at early prophase I (zygotene) display a strongly stained, large nucleolus and two more weakly stained, smaller bodies (Figure 1b). As division advances, all three nucleoli undergo size reduction and structural disorganization and part of the nucleolar components associates with chromosomal regions or lies along the length of the chromosomes (Figure 1c). During diplotene/diakinesis and metaphase I, and also at metaphase II, the nucleolar components are apparently distributed at the periphery in almost all chromosomes (Figure $1 \mathrm{~d}, \mathrm{e}$ ) (the chromosomal complement of this species is $2 \mathrm{n}=12 \mathrm{~A}+\mathrm{XY}$ ). At anaphase I and II and telophase, the nucleolar components are associated with two chromosome pairs (Figure 1f,g). In the round spermatid, there are two spherical Ag-stained bodies and another that is either elongate or rod-shaped (Figure 1h). During the formation of the spermatozoal tail, one of the round bodies is located at the anterior region of the head while the other and the rod-shaped body migrate together to the beginning of the posterior region (Figure 1i,j). The spermatids at a more advanced developmental stage, which already show a very elongate head, display two very evident, large, Ag-stained regions, occupying almost the entire head, separated from each other by a transverse band (Figure 1k,1). 


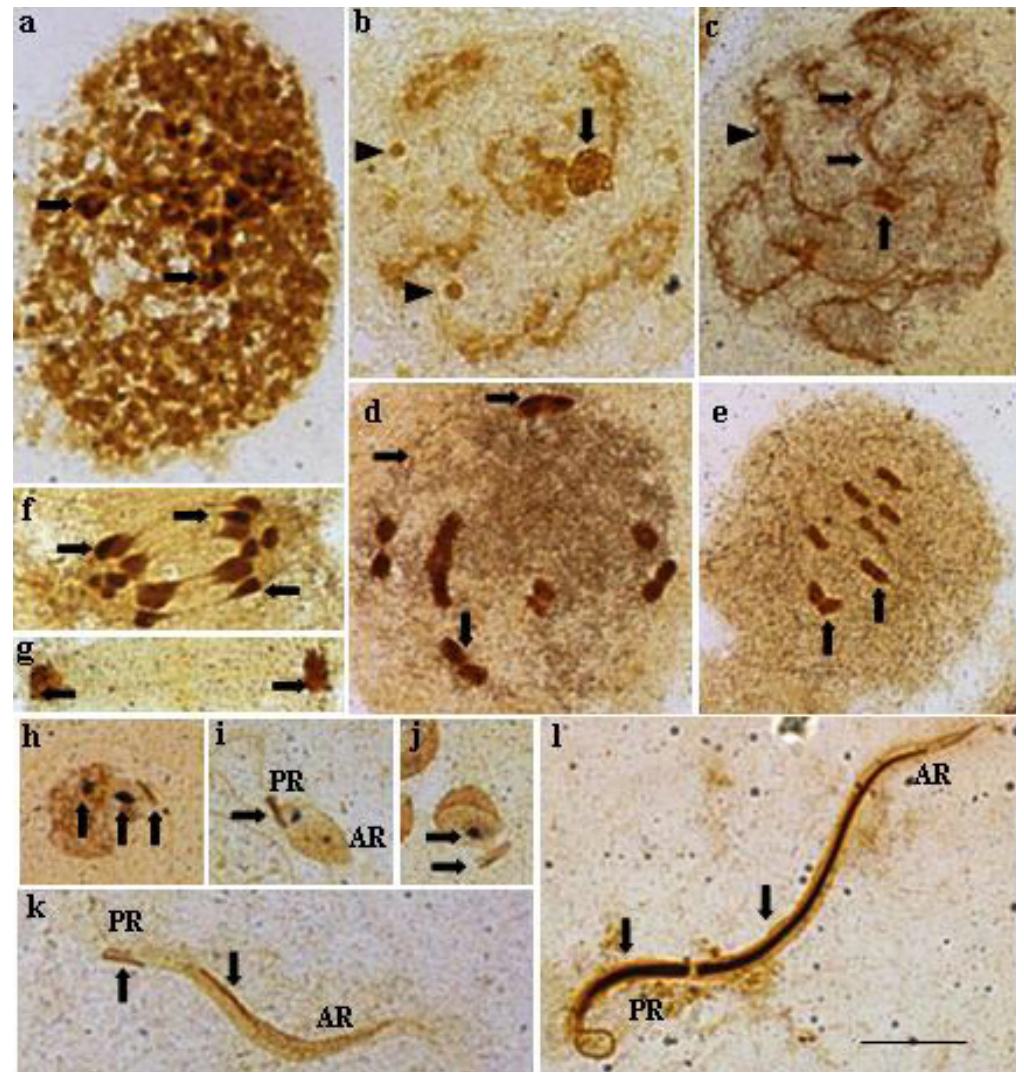

Figure 1. Silver nitrate-stained cells of Antiteuchus tripterus testicular lobes 1-3. (a) Polyploid nucleus with several silver-stained regions (arrows); (b) prophase I, zygotene - a larger, more evident nucleolus (arrow) and two smaller and not so evident nucleolar bodies (arrowheads); (c) leptotene - larger number of stainings at specific chromosomal regions (arrows) or smaller number binding along chromosomes (arrowhead); (d) diakinesis with silver staining at the periphery in the chromosome (arrows); (e) metaphases II - chromosomes showing silver staining (arrows); (f) anaphase I - chromosomes with silver staining at the periphery in the chromosome (arrows); (g) telophase beginning of nucleolar reorganization (arrows); (h) spermatid with two round and one rod-shaped nucleolar silver staining (arrows); (i-k) as the spermatid elongates, one round body localizes in the anterior region and the other round plus the rod-shaped bodies migrate to the posterior region of the spermatid head (arrows); (1) spermatids at a more advanced developmental stage display two regions separated from each other by a transverse band (arrows). $\mathrm{AR}=$ anterior region; $\mathrm{PR}=$ posterior region of the spermatid head. $\mathrm{Bar}=10 \mu \mathrm{m}$.

In lobe 4 , the polyploid nuclei show a large number of silver-impregnated bodies, more numerous and greater than in lobes 1-3 (Figure 2a). At the "diffuse" stage, which characterizes the prophase in spermatogonial cells in this lobe, large and strongly stained nucleolar masses are observed. As cell division advances, smaller Ag-stained regions are also seen in the chromosomes (Figure 2b,c). The round spermatids exhibit 4 strongly stained bodies and one larger region more weakly stained (Figure $2 \mathrm{~d}, \mathrm{e}$ ). In the elongate spermatids, the head is strongly and almost completely impregnated with silver. Later, the stained region subdivides into fragments of diverse sizes and spacing (Figure 2f,g). 


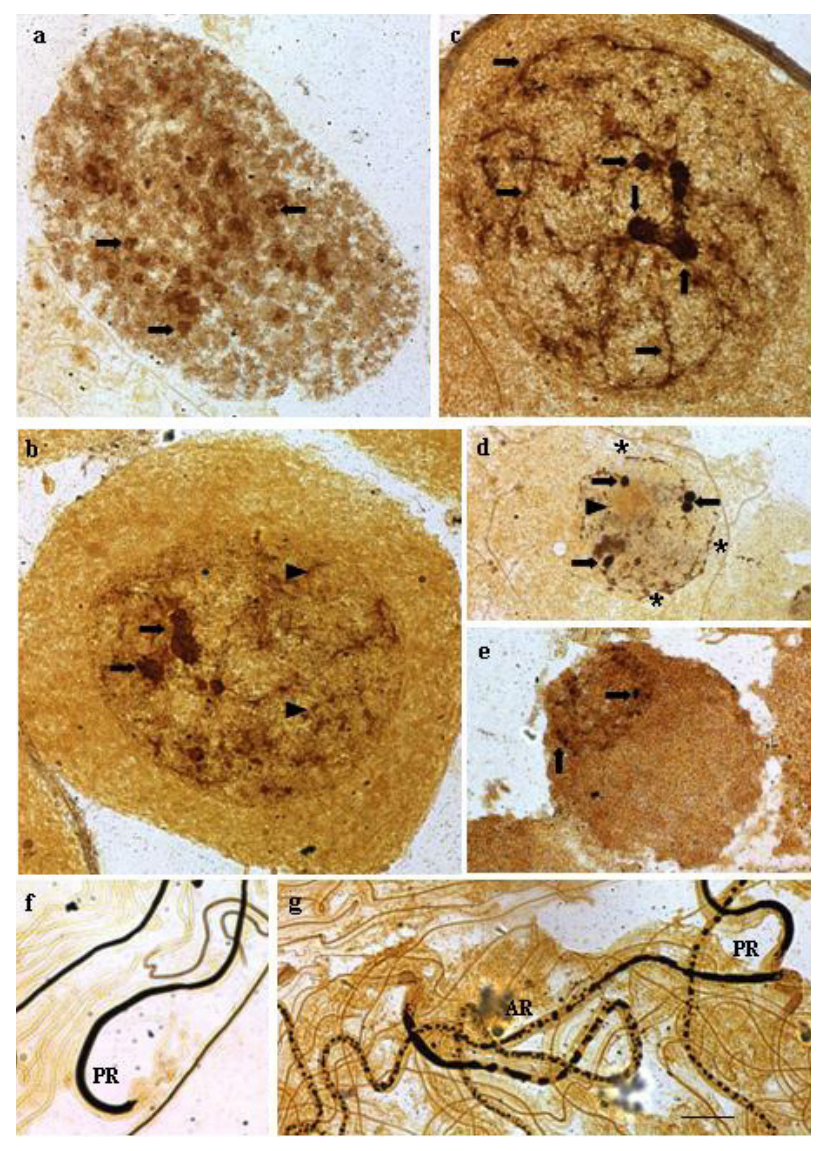

Figure 2. Silver nitrate-stained cells of Antiteuchus tripterus testicular lobe 4. (a) Polyploid nucleus with several silver-stained regions (arrows); (b, c) cells at "diffuse" stage evidencing strongly silver-stained regions (arrows) and silver staining along the chromosomes (arrowheads); (d, e) round-shaped spermatids showing strongly stained bodies (arrows) and one larger, weakly stained region (arrowhead), as well as small deposits along the chromosomes (asterisks); (f) anterior and posterior regions of the spermatid head are strongly stained; as development advances the stained anterior region subdivides, and that in the posterior region remains homogeneous $(\mathrm{g}) . \mathrm{AR}, \mathrm{PR}=$ anterior and posterior regions of the spermatid head, respectively. $\mathrm{Bar}=10 \mu \mathrm{m}$.

In lobe 5, the spermatogonial cells at prophase have large silver-stained masses present amid the decondensed chromosomes (Figure 3a). At metaphase I and anaphase I, filaments connecting stained chromatin masses can be observed (Figure 3b,c). The round spermatids formed in this lobe are of three different sizes (small, medium and large) as described by Souza et al. (2007). The number of nucleolar bodies varies based on spermatid size: smaller spermatids usually display one nucleolar body, while larger ones have four (Figure 3d). When the spermatid nucleus starts to elongate, two silver-stained bodies, one round and the other rod-shaped, are observed in the posterior region of the developing head (Figure 3e). Later, the three types of elongate spermatids exhibit a large-stained area in the anterior region of the head, separated from a smaller stained region located in the 
posterior region (Figure 3f). As development proceeds, this material is distributed in the anterior region of the spermatid showing an apparently coiled organization.
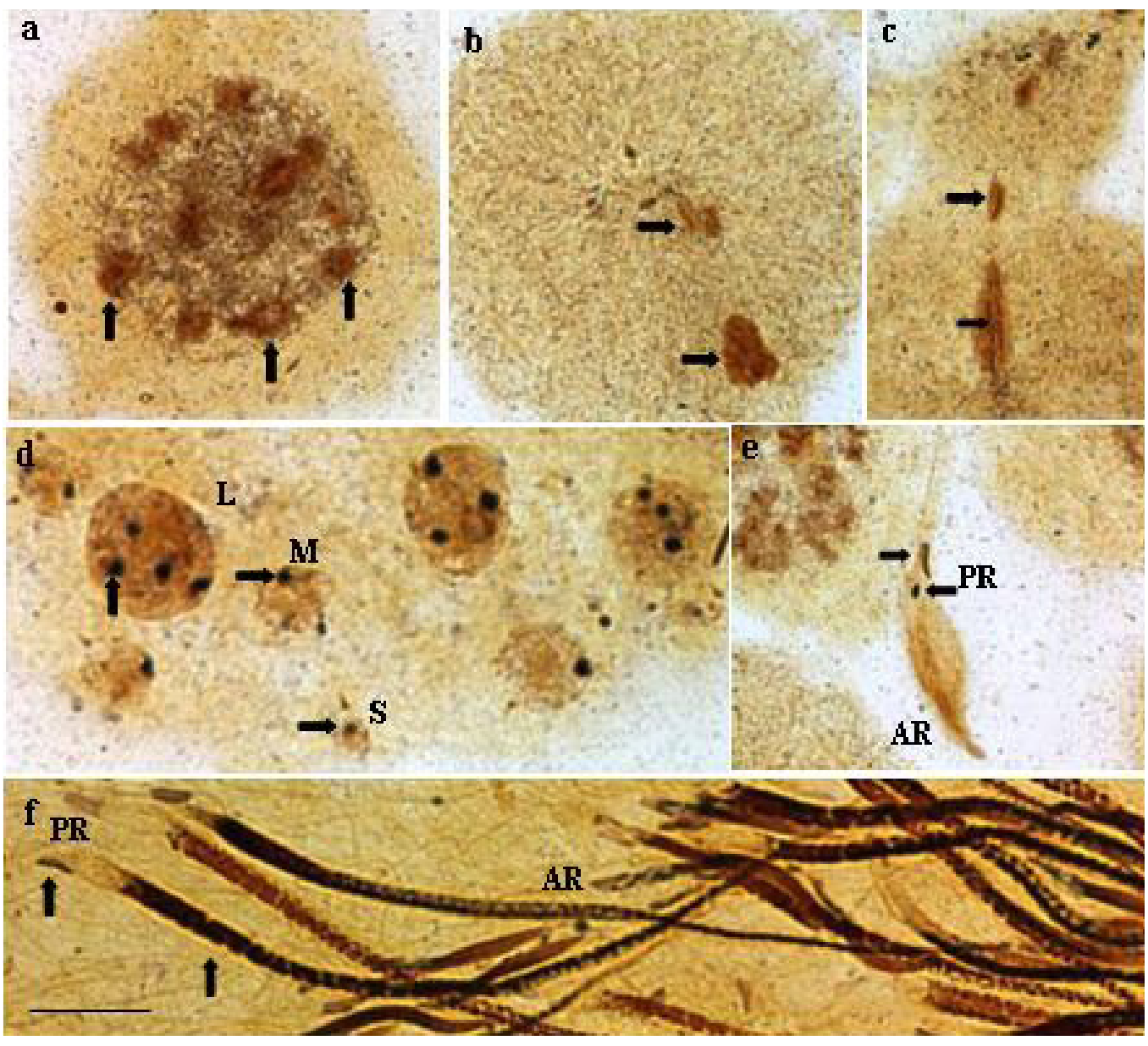

Figure 3. Silver nitrate-stained cells of Antiteuchus tripterus testicular lobe 5. (a) Cell at meiotic prophase I with silver-stained regions (arrows); (b) metaphase I with stained filaments in the chromatin mass (arrows); (c) anaphase I with silver-stained filaments joining chromatin masses (arrows); (d) small (S), medium (M) and large (L) roundshaped spermatids with a variable number of nucleoli (arrows); (e) one silver-stained round and one rod-shaped nucleolar body in the posterior region of the elongating spermatid head (arrows); (f) a heterogeneous-, large-stained area in the anterior region separated from a small-stained region located in the posterior region of the head (arrows). $\mathrm{AR}=$ anterior region; $\mathrm{PR}=$ posterior region of the spermatid head. $\mathrm{Bar}=10 \mu \mathrm{m}$.

In lobe 6, which also shows spermatogonial cells at the "diffuse" stage, nucleolar bodies have different sizes and are apparently associated with the dispersed chromatin (Figure 4a). The round spermatids are large and show two large, strongly stained nucleoli (Figure 4b). 
The elongate spermatids exhibit a large deposit of nucleolar material, which occupies all the interior of the head being discontinuous in its anterior region and continuous in the posterior region (Figure 4c,d).

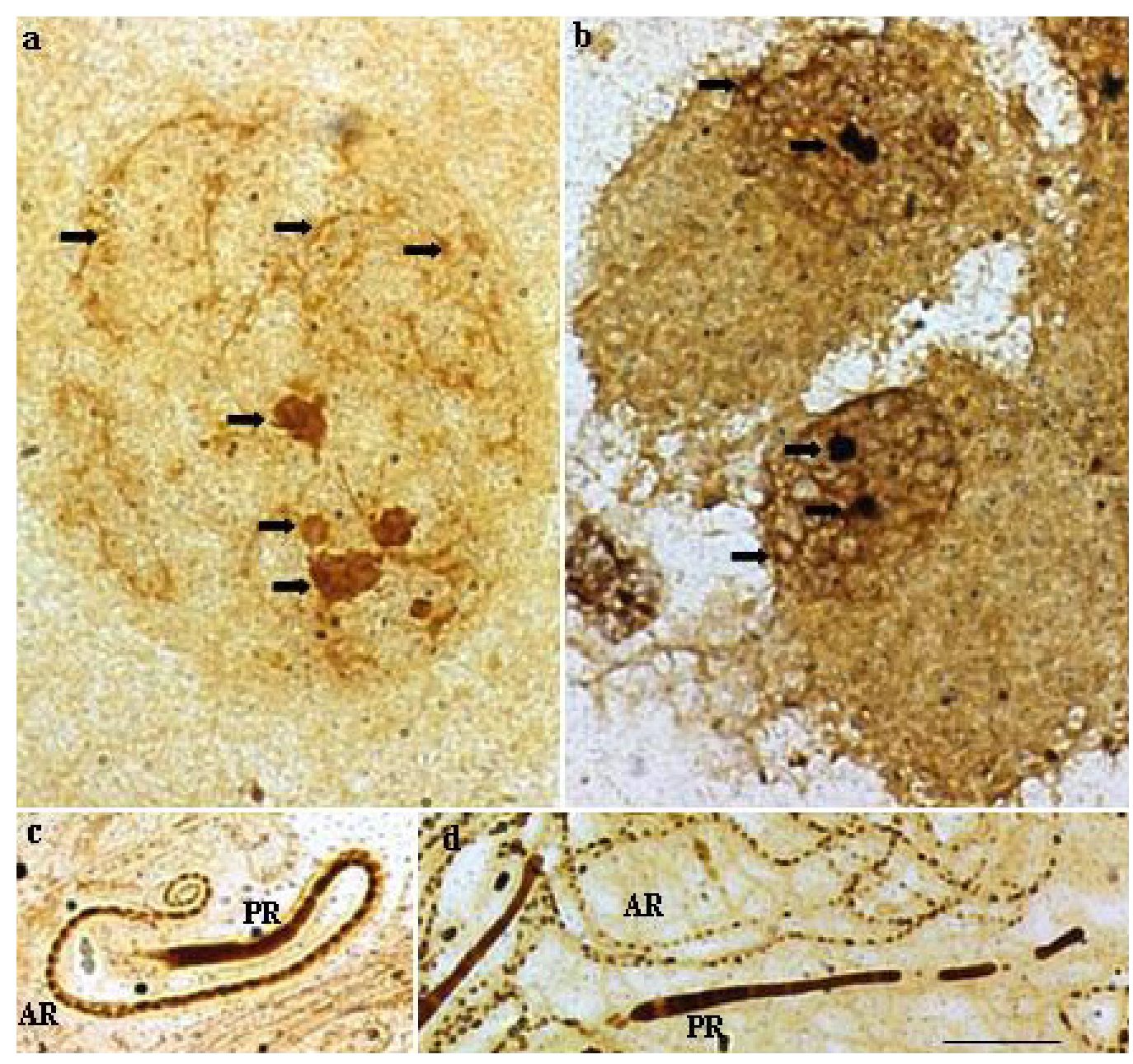

Figure 4. Silver nitrate-stained cells of Antiteuchus tripterus testicular lobe 6. (a) Cells at "diffuse" stage show 3 larger and 2 smaller nucleoli and silver impregnation along chromatin threads (arrows); (b) spermatids with 2 nucleoli and silver impregnation along the chromosomes (arrows); (c, d) elongate spermatids showing the posterior region with a homogeneous deposit of nucleolar proteins while a heterogeneous deposit is seen in the anterior region. $\mathrm{AR}=$ anterior region; $\mathrm{PR}=$ posterior region of the spermatid head. $\mathrm{Bar}=10 \mu \mathrm{m}$.

A summary of the variations in morphology and location of the stained nucleolar bodies among the testicular lobes are shown in Table 1. 
Ag-staining in testicular lobes of Antiteuchus tripterus

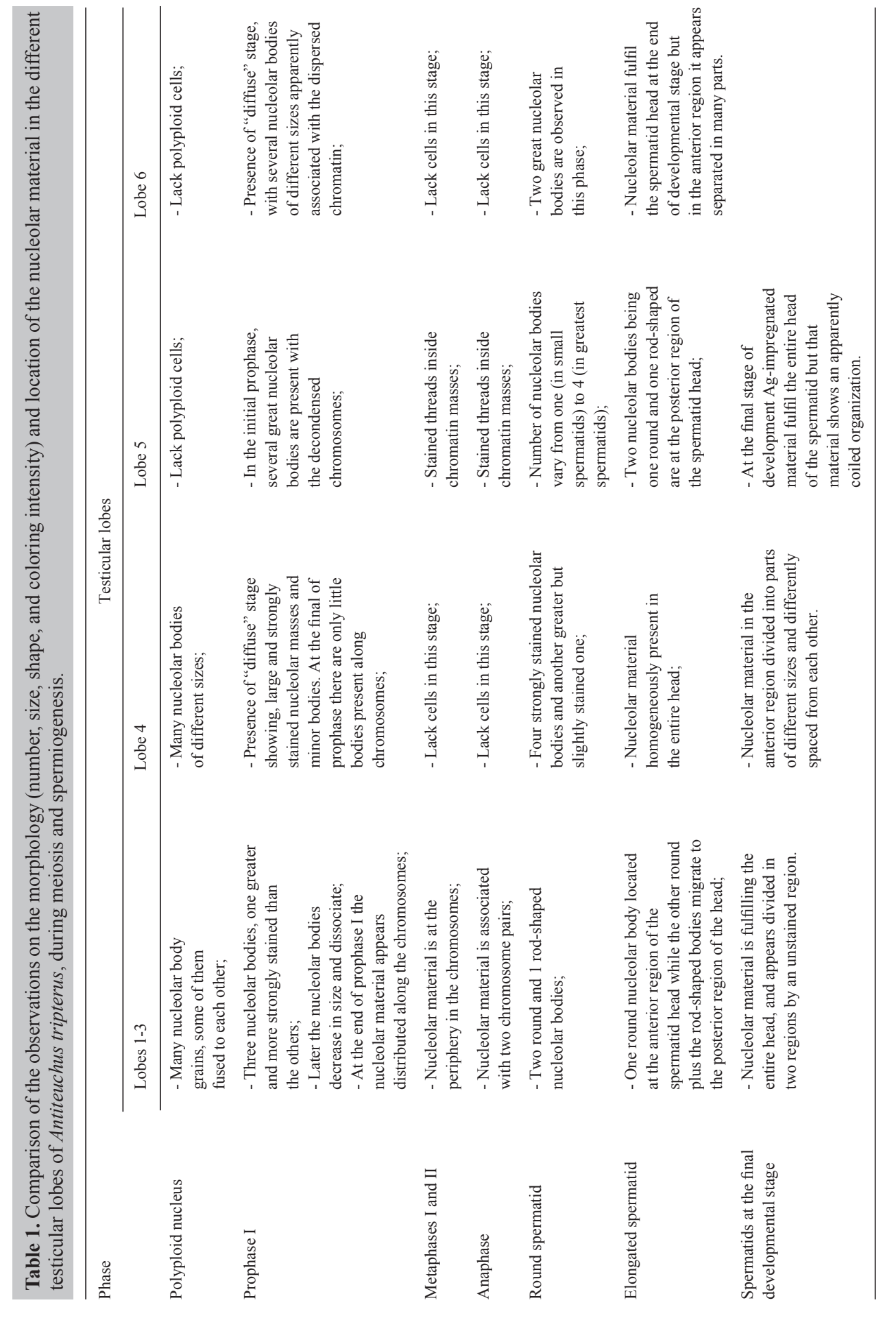

Genetics and Molecular Research 7 (1): 196-206 (2008) 


\section{DISCUSSION}

NORs are the chromosomal regions that associate with nucleoli and are responsible for their production. Ribosomal DNA that encodes rRNA is located in NORs (McClintock, 1934; Brown and Gurdon, 1964; Perry, 1965).

The Ag technique is normally used to stain NORs and nucleoli. However, sometimes NORs fail to stain. This was the case in the present study: silver-staining technique did not impregnate NORs. Thus, the number and chromosomal location of NORs could not be determined with certainty in A. tripterus. The number of nucleolar bodies is not a reliable indicator for this purpose, because the number of these bodies present in a cell may not correspond to the number of NORs, as two or more of these structures may associate to form a single nucleolar body (Ferguson-Smith and Handmarker, 1961). However, an indication was provided by the spermatogonial cells at early prophase I, in lobes 1-3 (the lobes which show the standard meiosis). In these lobes, a characteristic pattern of a large, well-defined nucleolus and two smaller nucleolar bodies is observed. As described in other insects, such as Drosophila, the large body could be the main nucleolus and the two smaller ones could be additional nucleoli produced by secondary NORs (Bicudo, 1982). Thus, it is possible that in A. tripterus, there is indeed a single NOR. This is also suggested by the stained region seen in two chromosomes at anaphase I, indicating that this single NOR is located in one autosomal pair, as has been previously reported in other Pentatomidae species.

The number and localization of NORs are known in only a few Heteropteran species and have been determined by different cytogenetic techniques. All the species described to date show only one NOR at the middle or subterminal portion of an autosome or sex chromosome (Camacho et al., 1985; Fossey and Liebenberg, 1995; González-Garcia et al., 1996; Papeschi and Bressa, 2002; Papeschi et al., 2003; Cattani et al., 2004). In Pentatomidae, a single NOR was detected at an autosomal telomeric region in Edessa meditabunda (Rebagliati et al., 2003), at the median portion of an autosome in Nezara viridula (Camacho et al., 1985; Papeschi et al., 2003), and at the telomeric region of the X chromosome in Graphosoma italicum (GonzálezGarcia et al., 1996).

Additionally, in the present study, the changes of Ag-stained material that took place during meiosis and spermiogenesis in the six testicular lobes could be observed. Variations include the number, size, shape, and distribution of nucleolar bodies. These features were similar in lobes 1-3. The remaining lobes, however, differed from each other and from lobes 1-3 as well. One of the differences is that in prophase cells the number and size of nucleolar bodies were greater in lobes 4 and 6 than in lobes 1-3. Due to the role played by the nucleolus in protein synthesis, this difference could be related to the function attributed to each lobe. While lobes 1-3 seem to be responsible for the production of spermatozoa involved in reproduction (Sivinski, 1980; Silberglied et al., 1984; Swallow and Wilkinson, 2002), lobes 4-6 seem to be involved in nutrition (Souza et al., 2007).

Another observation was that, in metaphase cells, nucleolar bodies are no longer visible but remain associated with the chromosomes at metaphases and anaphases in lobes 1-3 and 5 (in the remaining lobes, no cells were detected at these phases of the cell cycle). These observations indicate the persistence of partially organized nucleolar material during the entire meiosis in $A$. tripterus, a pattern that, to our knowledge, has not been described in meiotic chromosomes.

The differences among the testicular lobes as to the organization of the Ag-stained material in the elongate spermatids were striking, varying from an almost completely ho- 
mogeneous distribution, as observed in lobes 1-3, to a division in parts of different size and spacing, observed in lobes 4 and 6, or even displaying an apparently coiled organization, as in lobe 5. For now, we cannot explain the role of these variations. However, they and several of the other features described here encourage further investigations in the testicular lobes of A. tripterus in order to assess their functional meaning. In addition to the biological interest, the Pentatomidae include important agricultural pests for which an added knowledge of their biology may be helpful in the development of control methods.

\section{ACKNOWLEDGMENTS}

Special thanks go to Prof. Dr. Sonia Maria Oliani of the Department of Biology, IBILCE/UNESP for the opportunity to capture cell images, and to FAPESP, FUNDUNESP and $\mathrm{CNPq}$ for their financial support.

\section{REFERENCES}

Bicudo HEMC (1982). Silver staining and the nucleolar organizing activity in Drosophila species of the mulleri complex and their hybrids. Rev. Bras. Genet. 5: 31-50.

Bressa MJ, Papeschi AG, Fumagalli E, van Doesburg PH, et al. (2003). Cytogenetic and nucleolar meiotic cycle analyses in Dysdercus imitator Blote, 1931 (Pyrrhocoridae, Heteroptera) from Argentina. Folia Biol. 51: 135-141.

Brown DD and Gurdon JB (1964). Absence of ribosomal RNA synthesis in the anucleolate mutant of Xenopus laevis. Proc. Natl. Acad. Sci. USA 51: 139-146.

Camacho JPM, Belda J and Cabrero J (1985). Meiotic behaviour of the holocentric chromosomes of Nezara viridula (Insecta, Heteroptera) analyzed C-banding and silver impregnation. Can. J. Genet. Cytol. 27: 490-497.

Cattani MV, Greizerstein EJ and Papeschi AG (2004). Male meiotic behavior and nucleolus organizer regions in Camptischium clavipes (Fabr.) (Coreidae, Heteroptera) analyzed by fluorescent banding and in situ hybridization. Caryologia 57: 267-273.

Demerec M and Kaufmann BP (1945). Drosophila Guide. The Lord Baltimore Press, Baltimore.

Di Castro M, Prantera G, Cipriani A and Rocchi A (1983). Silver staining analysis of nucleolar-organizer activity during spermatogenesis of Asellus aquaticus (Crustacea, Isopoda). Genetica 60: 163-166.

Ferguson-Smith MA and Handmaker SD (1961). Observations on the satellited human chromosomes. Lancet 1: 638640.

Fossey A and Liebenberg H (1995). Meiosis and nucleolar structures in the stink bug Carlisis wahlbergi Stal (Coreidae: Heteroptera). Cytobios 81: 7-15.

González-Garcia JM, Antonio C, Suja JA and Rufas JS (1996). Meiosis in holocentric chromosomes: kinetic activity is randomly restricted to the chromatid ends of sex univalents in Graphosoma italicum (Heteroptera). Chromosome Res. 4: 124-132.

Goodpasture C and Bloom SE (1975). Visualization of nucleolar organizer regions in mammalian chromosomes using silver staining. Chromosoma 53: 37-50.

Howell WM and Black DA (1980). Controlled silver-staining of nucleolus organizer regions with a protective colloidal developer: a 1-step method. Experientia 36: 1014-1015.

Howell WM, Denton TE and Diamond JR (1975). Differential staining of the satellite regions of human acrocentric chromosomes. Experientia 31: 260-262.

McClintock B (1934). The relation of a particular chromosomal element to the development of the nucleoli in Zea mays. Z. Zellforsch. 21: 294-328.

Miller DA, Tantravahi R, Dev VG and Miller OJ (1977). Frequency of satellite association of human chromosomes is correlated with amount of Ag-staining of the nucleolus organizer region. Am. J. Hum. Genet. 29: 490-502.

Papeschi AG and Bressa MJ (2002). Cytogenetic studies in Belostomatidae from Argentina. In: Abstracts of the Second Quadrennial Meeting of the International Heteropterists Society, St. Petersburg, 46.

Papeschi AG, Mola LM, Bressa MJ, Greizerstein EJ, et al. (2003). Behaviour of ring bivalents in holokinetic systems: alternative sites of spindle attachment in Pachylis argentinus and Nezara viridula (Heteroptera). Chromosome Res. 11: 725-733. 
Perry RP (1965). The nucleolus and the synthesis of ribosomes. Natl. Cancer Inst. Monogr 18: 325-340.

Rebagliati P, Papeschi AG and Mola LM (2003). Meiosis and fluorescent banding in Edessa meditabunda and E. rufomarginata (Heteroptera: Pentatomidae: Edessinae). Eur. J. Entomol. 100: 11-18.

Santos J, Sentis C and Fernandez-Piqueras J (1987). Pattern of nucleolar organizer region activity during male meiosis in Callicrania seoanei (Orthoptera) as analyzed by silver staining: evidences for a possible reactivation in the period between the two meiotic divisions. Genome 29: 516-518.

Silberglied RE, Shepherd JG and Dickinson JL (1984). Eunuchs: the role of apyrene sperm in Lepidoptera? Am. Nat. 123: 255-265.

Sivinski J (1980). Sexual selection and insect sperm. Fla. Entomol. 63: 99-111.

Souza HV, Bicudo HEMC, Costa LAA and Itoyama MM (2007). A study of meiosis and spermatogenesis in different testicular lobes of Antiteuchus tripterus (Heteroptera, Pentatomidae). Eur. J. Entomol. 104: 353-362.

Swallow JG and Wilkinson GS (2002). The long and short of sperm polymorphisms in insects. Biol. Rev. Camb. Philos. Soc. 77: 153-182.

Tavares MG and Azeredo-Oliveira MTV (1997). Pattern of nucleolar activity during spermatogenesis in triatomines (Heteroptera, Reduviidae) as analyzed by silver staining. Cytobios 89: 93-103.

Yoshida T (1947). Unusual type of the nucleolus observed in a bug, Acanthocoris sordidus. J. Fac. Sci. Hokkaido Univ. (Zool.) 9: 243-249. 